\title{
Usefulness of Vessel Wall MR Imaging for Follow-Up after Stent-Assisted Coil Embolization of Intracranial Aneurysms
}

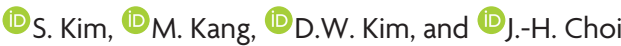

\begin{abstract}
BACKGROUND AND PURPOSE: Follow-up with MRA for intracranial aneurysms after stent-assisted coiling is complicated by imaging artifacts. We evaluated the usefulness of an alternative method: vessel wall MR imaging.
\end{abstract}

MATERIALS AND METHODS: We conducted a single-center, retrospective review of medical records of 47 patients who underwent 3D TOF-MRA, vessel wall MRI, and DSA after stent-assisted coiling between March 2016 and January 2018 . We evaluated the mean value of the signal intensity in the stented artery and the contralateral normal artery on vessel wall MRI. The quality of visualization was further compared between TOF-MRA and vessel wall MRI. Furthermore, we evaluated the diagnostic accuracy and concordance rate of TOF-MRA and vessel wall MRI for assessing the patency of the stented parent artery. DSA was used as a reference test.

RESULTS: The mean signal intensities of the stented and normal arteries on vessel wall MRI were not significantly different $(P=.133)$. The mean scores for the visualization of the stented parent artery on vessel wall MRI were significantly superior to those of TOF-MRA images $(P<.001)$. Vessel wall MRI reached an excellent positive predictive value $(100 \%)$. However, TOF-MRA had a poor positive predictive value (11\%; 95\% Cl, 9\%-12\%). The likelihood ratios of vessel wall MRI and TOF-MRA were $27.36(P<.001)$ and $2.98(P=.225)$, respectively. The concordance rate of vessel wall MRI and TOF-MRA with DSA for evaluating the state of the stented artery was $100 \%(\kappa=1)$ and $28 \%(\kappa=$ 0.038), respectively.

CONCLUSIONS: Vessel wall MRI may be useful in evaluating the patency of stented arteries after stent-assisted coil embolization for intracranial aneurysms.

ABBREVIATIONS: $\mathrm{SI}=$ signal intensity; $\mathrm{VWMRI}=$ vessel wall MRI

$E^{n}$ ndovascular coil embolization has become an established treatment method for intracranial aneurysms. ${ }^{1}$ Intracranial stents are used to treat wide-neck aneurysms using the neck-remodeling technique. ${ }^{2}$ However, stents cause endothelial hyperplasia and thrombosis, which can lead to stenosis or stented artery occlusion. The incidence of in-stent stenosis is approximately $1 \% .^{3,4}$ In-stent thrombosis may be more common-reported to be as high as $4 \%$ in 1 series - and may account for as much as $29 \%$ of thromboembolic complications. ${ }^{3}$ The criterion standard for evaluating this complication and the outcome of aneurysm treatment is DSA. There has been a move toward noninvasive fol-

Received April 15, 2018; accepted after revision July 30.

From the Departments of Radiology (S.K., M.K., D.W.K.) and Neurosurgery (J.-H.C.), Dong-A University Medical Center, Busan, Korea.

Please address correspondence to Myongjin Kang, MD, Department of Radiology, Dong-A University Medical Center, 1,3-ga, Dongdaeshin-dong, Seo-gu, Busan 602-715, Korea; e-mail: myongjinkang1@gmail.com

Indicates article with supplemental on-line photo.

http://dx.doi.org/10.3174/ajnr.A5824 low-up using MR imaging due to the risks inherent in the invasive DSA procedure.

A widely used noninvasive alternative to DSA is $3 \mathrm{D}$ TOFMRA. ${ }^{5}$ However, it is difficult to interpret the patency and/or degree of stenosis on TOF-MRA because of susceptibility artifacts and radiofrequency shielding of intracranial nitinol stents. ${ }^{6}$ Contrast-enhanced MRA has been reported to yield better results than TOF-MRA for the evaluation of aneurysm occlusions, but the ability of contrast-enhanced MRA to evaluate stented-artery status remains unclear. ${ }^{7}$ Furthermore, the use of contrast materials in contrast-enhanced MRA is associated with anaphylactic shock and nephrogenic systemic fibrosis; the frequency of all acute, adverse events after an injection of 0.1 or $0.2 \mathrm{mmol} / \mathrm{kg}$ of gadolinium chelate ranges from $0.07 \%$ to $2.4 \%{ }^{8}$ To reduce this risk, we attempted to find a useful imaging technique to evaluate the stented parent artery as an alternative to both contrast agents and DSA.

Vessel wall MR imaging (VWMRI) has been increasingly used to study intracranial vascular lesions, such as atherosclerosis, vasculitis, and aneurysms. ${ }^{9}$ In practice, the signal loss caused by the 
stent on TOF-MRA makes evaluation of the parent artery difficult. However, VWMRI is closely related to fast spin-echo techniques with variable flip angle refocusing pulses, which use radiofrequency refocusing pulses to return a T2' decay of transverse magnetization to a longer T2 decay, thus reducing signal loss. ${ }^{10}$ We hypothesized that even without contrast administration, VWMRI could provide good image quality for evaluating stented parent arteries. Thus, the present study evaluated the usefulness of VWMRI after stent-assisted coil embolization for intracranial aneurysms and compared its efficacy with that of TOF-MRA.

\section{MATERIALS AND METHODS \\ Patients}

This study was approved by our institutional review board, and the requirement for written informed consent was waived. We retrospectively reviewed the medical records of patients who had an intracranial aneurysm treated by stent-assisted coiling between March 2016 and January 2018. Before patient discharge, our institution performs an initial MR imaging with TOF-MRA and VWMRI after stent-assisted coil embolization. Follow-up is performed by MR imaging with TOF-MRA and VWMRI. Conventional angiography is performed if there is suspicion of aneurysm recanalization or stent-related complications (eg, thromboembolism, stenosis, or occlusion).

\section{MR Imaging Protocol}

MR imaging was performed with a 3T MR imaging system (Discovery MR750; GE Healthcare, Milwaukee, Wisconsin) using a 32-channel head coil. The VWMRI consisted of 3D fast spinecho proton-density imaging with variable flip angles (Cube; GE Healthcare) and motion-sensitized driven equilibrium. The imaging parameters for $3 \mathrm{D}$ proton-density images were as follows: TR, 1500 ms; TE, $21.3 \mathrm{~ms}$; bandwidth, $62.5 \mathrm{kHz}$; FOV, $190 \times$ $190 \mathrm{~mm}$; matrix, $384 \times 384$; in-plane resolution, $0.49 \times 0.49 \mathrm{~mm}$; slice thickness, $0.8 \mathrm{~mm}$ (interpolated to $0.4 \mathrm{~mm}$ ); NEX, 1; Autocalibrating Reconstruction for Cartesian imaging acceleration factor, 1.5; scan time, 7 minutes, 53 seconds. We obtained 3D TOF-MRA with the following parameters: TR, $23 \mathrm{~ms}$; TE, $2.5 \mathrm{~ms}$; flip angle, $20^{\circ}$; bandwidth, $50 \mathrm{kHz}$; FOV, $210 \times 180 \mathrm{~mm}$; matrix, $416 \times 224$; slice thickness/slice overlap, $1.2 \mathrm{~mm} / 16 \%$; 3 slabs using multiple overlapping thin-slab acquisition; asset factor, 2; scan time, 4 minutes, 32 seconds. For 3D TOF-MRA, maximum-intensity-projection reconstructions and source images were used. For VWMRI, minimum-intensity-projection reconstructions and source images were used.

\section{DSA}

Intra-arterial DSA was performed with a biplane neuroangiographic system (AXIOM Artis dBA; Siemens, Erlangen, Germany). Using transfemoral catheterization, selective injections of the internal carotid or vertebral arteries were performed according to the aneurysm location. All DSA examinations included anteroposterior, lateral, and working views.

\section{Image Analysis}

We performed quantitative analyses of relative signal intensity (SI) and visualization of the stented parent arteries on the images obtained by VWMRI after the procedures.
One observer manually drew an ROI of at least $1 \mathrm{~mm}^{2}$ on the single-slice source images (On-line Figure). An ROI was placed at the stented artery adjacent to the aneurysm neck on the basis of the assumption that an aneurysm would be located in the middle of the stented artery in most cases because we consistently placed the center of the stent at the aneurysm neck site during stentassisted coiling. DSA and VWMRI are different imaging modalities, and it is difficult to match the exact position of the stent by comparing the VWMRI with DSA. Moreover, the stent strut is not visible on VWMRI.

The mean value of the SI in the stented artery was compared with that in the contralateral normal artery. The relative SI of the stented artery was calculated as follows: SI of the Stented Artery/SI of the Contralateral Normal Artery. In the case of the basilar artery, the proximal normal artery was referred to as the contralateral normal artery.

The image quality of the stented parent artery was graded subjectively on a 3-point scale: 1 = poor (evaluation could not be made), 2 = moderate (evaluation could be made but information was lacking compared with the DSA), and $3=$ good (image could be evaluated similar to DSA). ${ }^{11}$

Furthermore, we evaluated the status of the stented parent artery by comparing the VWMRI, TOF-MRA, and the DSA. The patency status of the parent artery was evaluated on a 3-point scale: 1 = patent (no change in the parent artery diameter), $2=$ stenosis (narrowing of the parent artery), 3 = parent artery occlusion. A simplified 2-grade scale was used to assess the diagnostic accuracies for parent vessel patency: normal and pathologic (stenosis, occlusion, or thrombosis).

Two interventional neuroradiologists (one with 10 years of experience and the other with 5 years) independently evaluated all images in a random order. In case of disagreement, a third experienced neuroradiologist with 17 years of experience helped to establish consensus. DSA findings were considered the standard reference and were interpreted independently by 1 neurosurgeon who was unaware of the MR imaging findings.

\section{Statistical Analysis}

A Wilcoxon signed rank test was used to analyze the subjective scores for the quality of the stented artery images. $P$ values $<.05$ were considered statistically significant. The Mann-Whitney $U$ test was used to compare the mean relative SI value for the stented arteries with those of the contralateral normal arteries. The weighted $\kappa$ statistic was used to evaluate interobserver agreement for each technique and the concordance rate for evaluating the patency of the parent vessel using the results of DSA as a standard of reference. Statistical analyses were performed with MedCalc for Windows, Version 18.2.1 (MedCalc Software, Mariakerke, Belgium). Diagnostic accuracy was calculated with SPSS software, Version 11.5 (IBM, Armonk, New York).

\section{RESULTS}

Before discharge, 84 patients underwent stent-assisted coiling and an initial MR imaging; 2 patients were excluded from our study because the intraluminal signal intensity was too high, even in normal arteries, due to motion or flow artifacts; and 35 patients were excluded because the follow-up DSA was not available after 
discharge. The sample of the present study, therefore, totaled 47 patients (female/male ratio, 33:14; mean age, $57.2 \pm 8.9$ years; age range, 39-78 years) who underwent TOF-MRA, VWMRI, and DSA after their respective procedures. A Neuroform 3 stent (Stryker Neurovascular, Kalamazoo, Michigan) was used for 31 aneurysms, while a Solitaire AB stent (Covidien, Irvine, California) was used for 16 . The clinical characteristics of the patients are shown in Table 1.

All 47 parents underwent both follow-up MR imaging and DSA with an interval gap of $<7$ days. The mean period between coil embolization and follow-up DSA was $266 \pm 98$ days; the interval between the follow-up MR imaging and follow-up DSA was $3.3 \pm 1.8$ days.

\section{Relative Signal Intensity in the Stent}

We performed quantitative analyses of relative SI of the arteries in the images obtained by initial VWMRI after the procedures in 46 of the 47 patients; 1 patient was excluded due to signal intensity contamination in the parent artery by acute in-stent thrombosis. The mean SIs for the stented artery and normal artery were $167 \pm$ 20 and $174 \pm 20$, respectively, and were not significantly different $(P=.133)$. The mean relative SIs for the stented arteries using the Neuroform and Solitaire stents was $0.97 \pm 0.04$ and $0.94 \pm 0.05$, respectively; the mean relative SI of the stented artery fitted with Solitaire stents was lower than that of stented arteries fitted with Neuroform stents $(P<.013)$.

Table 1: Characteristics of the 47 patients and 47 aneurysms that underwent stent-assisted coiling

\begin{tabular}{lc}
\multicolumn{1}{c}{ Characteristics } \\
\hline Mean age (range) (yr) & $57.2 \pm 8.9(39-78)$ \\
Sex (female/male) & $33: 14$ \\
Ruptured aneurysms & $6(13 \%)$ \\
Location & 27 \\
$\quad$ Internal carotid artery & 9 \\
$\quad$ Middle cerebral artery & 5 \\
$\quad$ Anterior cerebral artery/anterior & \\
$\quad$ communicating artery & \\
$\quad$ Vertebrobasilar artery & 6 \\
Aneurysm size & \\
$\quad<5$ mm & 24 \\
$\quad 5-10$ mm & 21 \\
$\quad$ 10 mm & 2 \\
Initial occlusion result & \\
$\quad$ Complete occlusion & 38 \\
Remnant neck & 7 \\
$\quad$ Residual aneurysm & 2 \\
Stent & \\
$\quad$ Neuroform & 31 \\
Solitaire & 16 \\
\hline
\end{tabular}

\section{Image Quality}

For the Neuroform stent cases, the image quality of the stented parent artery on VWMRI was significantly superior to that obtained by TOF-MRA $(P<.001)$. The average scores were $3 \pm 0$ for VWMRI and $2.2 \pm 0.69$ for MRA. In cases of Solitaire stents, the image quality was equally poor for the stented parent artery on VWMRI and MRA. A proximal marker near the detachment zone of the Solitaire stent caused significant signal loss of the lumen due to susceptibility artifacts in both imaging modalities. When the evaluations were performed in the Solitaire stents and the proximal portion of the stented artery was excluded, the mean scores for the stented artery were $3 \pm 0$ and $1.7 \pm 0.7$ for VWMRI and TOF-MRA, respectively $(P<.001)$. Therefore, the quality for the stented artery images obtained by VWMRI was significantly superior to that observed in 3D TOF-MRA images. With the exception of the proximal portion in the Solitaire stent, this finding was consistent regardless of the type of stent used. The overall mean scores for image quality, in terms of visualization of the stented artery, were $3 \pm 0$ and $2 \pm 0.7$ for VWMRI and 3D TOF-MRA, respectively $(P<.001)$.

\section{Patency of the Parent Vessel}

It was difficult to evaluate the patency of the parent artery near the detachment zone in Solitaire stents due to signal loss on VWMRI and MRA. However, apart from the marker region in the Solitaire stent, VWMRI showed results consistent with those of DSA. The statuses of the parent vessel artery assessed with VWMRI, TOFMRA, and DSA are shown in Table 2.

Interobserver agreement was very good for VWMRI $(\kappa=1)$ and good for TOF-MRA $(\kappa=0.64)$. The concordance rate of VWMRI and TOF-MRA with DSA for evaluating the state of the stented artery was $100 \%(\kappa=1)$ and $28 \%(\kappa=0.038)$, respectively. The likelihood ratios of VWMRI and TOF-MRA were $27.36(P<.001)$ and $2.98(P=.225)$, respectively. By means of the simplified 2-grade scale (normal-versus-pathologic vessels), VWMRI reached an excellent positive predictive value (100\%). However, TOF-MRA featured a poor positive predictive value (11\%; 95\% CI, 9\%-12\%). False stenosis was prevalent on TOF-MRA. Normal vessels on DSA were overestimated as pathologic vessels on TOF-MRA in 34/47 patients.

There were 4 cases of stent-related complications. An in-stent thrombus occurred after stent-assisted coiling for a paraclinoid ICA aneurysm (Fig 1). According to the 3-point scale, this case was classified as a stenosis, but 2 readers interpreted it as a thrombus on VWMRI. An angiogram obtained immediately after the final coil insertion revealed the acute small in-stent thrombosis. A 30-minute-delayed DSA performed after rescue use of tirofiban demonstrated no thrombus growth and uncompromised distal arterial flow velocity. Thus, we decided to follow-up without further interventional treatment. VWMRI revealed that the thrombosis had increased in size in the stent 2 days after the procedure. However, TOF-MRA showed luminal narrowing of the stented parent artery. Immediate, same-day follow-up DSA confirmed the thrombus growth in the stent, consistent with the VWMRI findings. The other stent-re-
Table 2: Concordance rate
a standard of reference

\begin{tabular}{|c|c|c|c|c|c|c|}
\hline & \multicolumn{3}{|c|}{ Vessel Wall MRI } & \multicolumn{3}{|c|}{ MRA } \\
\hline & Patent & Stenosis & Occlusion & Patent & Stenosis & Occlusion \\
\hline \multicolumn{7}{|l|}{ DSA } \\
\hline Patent & 43 & 0 & 0 & 9 & 29 & 5 \\
\hline Stenosis & 0 & 4 & 0 & 0 & 4 & 0 \\
\hline Occlusion & 0 & 0 & 0 & 0 & 0 & 0 \\
\hline Concordance rate & \multicolumn{3}{|c|}{$100 \%(\kappa=1)$} & \multicolumn{3}{|c|}{$28 \%(\kappa=0.038)$} \\
\hline
\end{tabular}



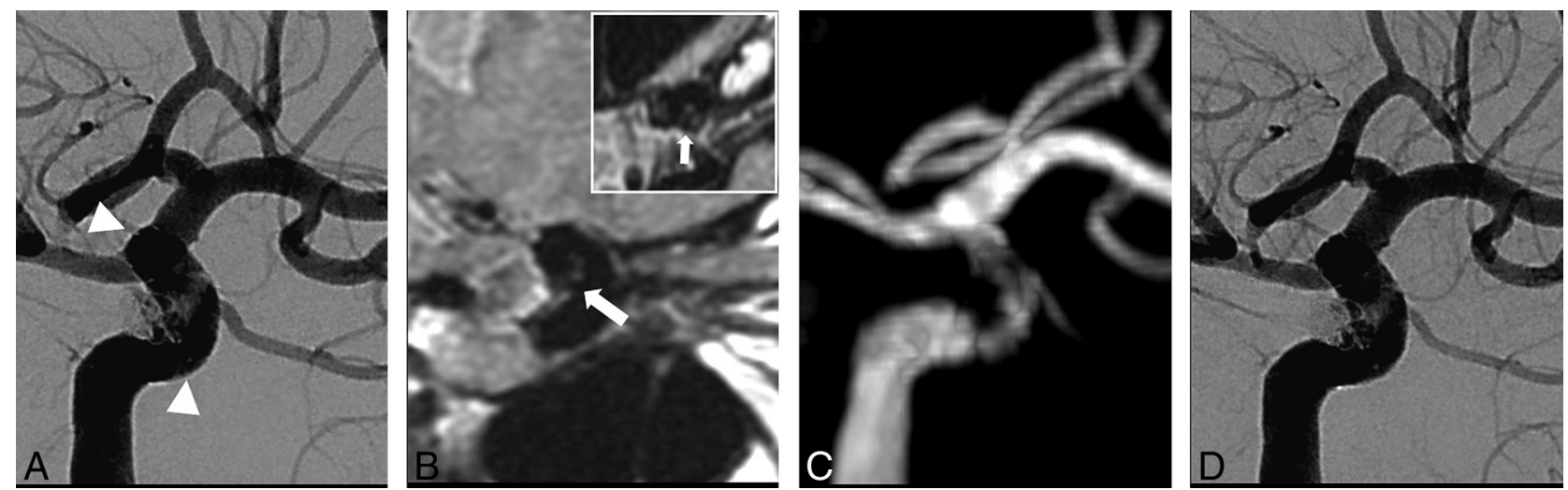

FIG 1. A 60-year-old woman with a paraclinoid internal carotid artery aneurysm. A, Cerebral angiography performed immediately after stentassisted coiling shows an acute in-stent thrombus. The arrowheads indicate the proximal and distal markers of the stent. $B$, Oblique and axial (upper right image) reformatted images of the proton-density image obtained from a vessel wall MR imaging performed 2 days after coiling reveal the in-stent thrombus. C, Time-of-flight MR angiography demonstrates artifactual luminal narrowing of the stented parent artery. $D$, Cerebral angiography on the same day shows the thrombus within the stent, consistent with vessel wall MR imaging results.
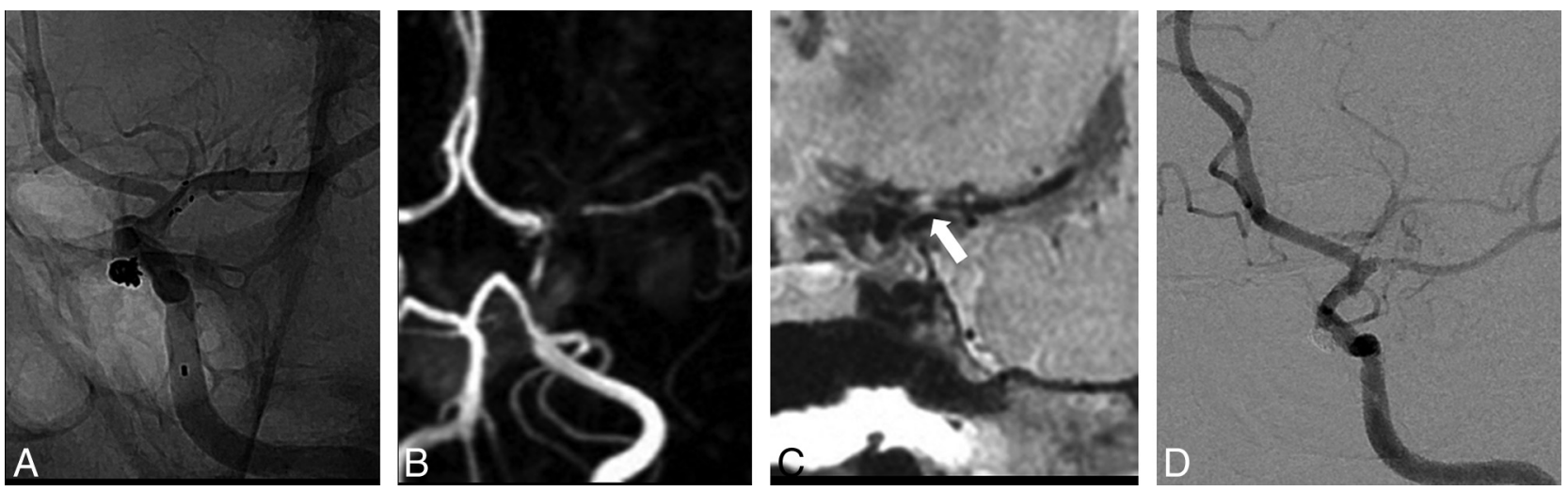

FIG 2. A 50-year-old man with a paraclinoid internal carotid artery aneurysm. A, Cerebral angiography performed immediately after stentassisted coiling shows the normal parent artery. B, Time-of-flight MR angiography obtained at a 1-year follow-up examination shows signal drop-out with a decreased diameter and signal intensity of distal vessels in the left middle cerebral artery. C, Oblique reformatted images of vessel wall MR imaging reveal stenosis in the left proximal middle cerebral artery (arrow). D, Follow-up cerebral angiography confirms stenosis of the left proximal middle cerebral artery.

lated complication observed in 3 cases was in-stent restenosis following stent-assisted coiling. VWMRI assisted in the identification of the 3 stenosis cases (Fig 2). All 3 cases were also classified as pathologic vessels on TOF-MRA, but we were unable to determine whether this represented artifactual luminal narrowing or a true lesion.

\section{Additional Results}

In our preliminary study, we evaluated coiled aneurysm stability with VWMRI. On the basis of the evolution of blood clots, the signal of clots decreases in the chronic phase on T1WI. Even in the chronic phase, the SI of hematoma is higher than the dark SI of blood. ${ }^{12}$ Thus, we hypothesized that SI in the coiled aneurysm would appear higher than that in the parent artery and that the residual flow signal in the coiled aneurysm would appear dark on the proton-density image. We therefore sought to evaluate aneurysm stability with VWMRI using SI differences between treated aneurysms and residual flow. Of the 47 patients, follow-up DSA results showed complete occlusion in 42 patients, a remnant neck in 4 patients, and residual aneurysm in 1 patient. Regarding the depiction of residual flow in the coiled aneurysms, the concordance rate of VWMRI was lower than that of TOF-MRA $(38 \%$ versus $96 \%$ ). In 42 cases of complete occlusion identified via DSA, 25 were erroneously interpreted as residual neck or residual aneurysm on VWMRI. In 2 patients whose TOF-MRA results were underestimated relative to DSA, a tiny residual neck was erroneously interpreted as complete occlusion.

\section{DISCUSSION}

An intracranial stent is a powerful tool for the endovascular treatment of wide-neck and fusiform aneurysms. However, stent-related complications can cause permanent neurologic complications. Identifying these complications is important for patient diagnosis, management, and prognosis. Although DSA is standard for the follow-up of coiled aneurysms, it is invasive and exposes patients to procedure-related complications, such as cerebral thromboembolism, contrast nephrotoxicity, and radiation. ${ }^{13}$ Thus, follow-up imaging after endovascular treatment is vital. We evaluated the usefulness of VWMRI after stent-assisted coil embolization of intracranial aneurysms. The primary finding of our study was that VWMRI could reliably identify in-stent complications.

First, we evaluated the relative SI of the stented parent arteries 
on VWMRI after performing stent-assisted coiling with 2 types of intracranial nitinol stents: Neuroform and Solitaire. The signal intensity of the stented artery was different according to stent type. The relative SI for stented arteries fitted with Neuroform stents was higher than that for the stented arteries fitted with Solitaire stents in our VWMRI study. These findings are consistent with an in vivo study that used a silicone tube to reveal that the mean relative in-stent signal for Neuroform stents was higher than that for the Solitaire stents. ${ }^{14}$ The extent of stent-induced signal loss on TOF-MRA has been shown to depend on the thickness of the strut and cell design. ${ }^{14}$ The Neuroform stent has an open-cell design and a strut thickness of $65 \mu \mathrm{m}$, while the Solitaire stent has a closed cell design and a thickness of $60 \mu \mathrm{m}$. Open-celldesign stents have been shown to have lower susceptibility to artifacts compared with closed-cell stents. ${ }^{14}$ Moreover, the partially overlapping strut in the Solitaire stent may cause more in-stent signal reduction than in Neuroform stents.

Second, we evaluated the image quality of the stented artery on VWMRI versus TOF-MRA. An intracranial stent can compromise the evaluation of the patency of the vessel on TOF-MRA. ${ }^{15}$ Contrast administration can improve vessel lumen visualization. ${ }^{16,17}$ Lövblad et al $^{16}$ assessed 19 patients with stent-assisted coil embolization using noncontrast TOF-MRA and contrast-enhanced TOF-MRA with 1.5T MR imaging; the contrast administration allowed better visualization of the vessel lumen in the cases that used nitinol stents. Furthermore, the in-stent signal loss was present in all TOF-MRA cases except for contrast-enhanced MRA images. Thamburaj et $\mathrm{al}^{17}$ assessed 42 patients with stent-assisted coiling and found that contrast-enhanced MRA showed a tendency to demonstrate superior in-stent flow, smoother margins, and minimal-to-no stenosis relative to noncontrast TOF-MRA. However, although complications of contrast agent administration for contrast-enhanced MRA are rare, contrast dye can cause severe allergic reactions and adverse effects. To avoid administration of contrast, we compared VWMRI with TOF-MRA: VWMRI provided better image quality compared with TOF-MRA. The latter method is based on a gradient-echo sequence. Image artifacts due to metal are caused by inhomogeneity in the static magnetic field, and gradient-echo-based sequences are therefore more prone to these artifacts. In contrast, VWMRI is based on a spinecho sequence, and its use of $3 \mathrm{D}$ techniques allows very thin slices; compared with thin 2D slices, susceptibility artifacts are reduced because of the (fast) spin-echo technique. ${ }^{9}$ In contrast to cases in which Neuroform stents were used, neither TOF-MRA nor VWMRI could evaluate the segment near the proximal marker of Solitaire stents. This issue is because the relatively thick, proximal marker near the detachment zone of the Solitaire stent caused complete signal loss of the lumen due to the susceptibility artifacts. However, when the proximal marker was excluded, the image quality of the stented artery on VWMRI was superior to that of TOF-MRA. Agid et al ${ }^{18}$ reported a "marker band effect" appearing in arteries $\leq 2 \mathrm{~mm}$ in diameter. The markers of Neuroform stents are made of platinum, which creates strong magnetic susceptibility artifacts. We observed this effect on TOF-MRA in cases in which the Neuroform stent was used in the middle cerebral or posterior cerebral artery. However, this phenomenon did not occur on VWMRI.
Third, we compared VWMRI and TOF-MRA with DSA to evaluate the status of the stented parent artery. Several studies found that MRA with or without contrast material does not seem to provide an effective alternative to DSA for assessing parent artery patency. ${ }^{18-21}$ In a direct comparison between contrast-enhanced MRA and DSA in 27 patients with 28 aneurysms, Agid et $\mathrm{al}^{18}$ reported 6 cases of false stenosis and 2 of exaggerated stenosis on contrast-enhanced MRA, suggesting that the contrast-enhanced MRA did not depict the true status of the artery. Marciano et $\mathrm{al}^{19}$ compared TOF-MRA and contrast-enhanced MRA with DSA in 33 patients with 35 aneurysms treated by stent-assisted coiling. The intermodality agreement was poor for 3D TOF-MRA $(\kappa=0.12)$ and null for contrast-enhanced MRA $(\kappa=-0.01)$. TOF-MRA $(\kappa=0.05)$ and contrast-enhanced MRA $(\kappa=-0.04)$ were not able to detect pathologic vessels better than DSA: No difference in accuracy was found $(P=.68)$. Recently, Akkaya et $\mathrm{al}^{20}$ evaluated the usefulness of contrast-enhanced MRA and TOF-MRA in 24 aneurysms treated with low-profile stent-assisted coiling. Although interobserver agreement was substantial in both TOF-MRA $(\kappa=0.71, P<.001)$ and contrast-enhanced MRA $(\kappa=0.64, P=.001)$, intermodality agreement values of TOF-MRA and contrast-enhanced MRA with DSA were insignificant in terms of stent patency $(\kappa=0.065, P=.27)$ and contrastenhanced MRA $(\kappa=0.158, P=.15)$. Choi et $\mathrm{al}^{21}$ compared 4D-MRA and 3D TOF-MRA in a group of 26 patients with aneurysms treated with stents. Although the visualization of the stented arteries on 4D-MRA was significantly superior to that on 3D TOF-MRA $(P<.001)$, the investigators doubted whether the degree of in-stent restenosis was accurately measured by $4 \mathrm{D}$ MRA. The ability of 4D-MRA to evaluate in-stent stenosis thus remains undetermined.

Recent studies have demonstrated that Silent MRA (Discovery MR750w; GE Healthcare) might be useful for follow-up imaging after stent-assisted coil embolization. ${ }^{22,23}$ In Silent MRA, an ultrashort TE can minimize the phase dispersion of the labeled blood flow signal in the voxel and decrease magnetic-susceptibility artifacts; accordingly, the artifacts from stents or coils are theoretically decreased. ${ }^{23}$ Thus, Silent MRA could improve visualization of the stent without contrast administration more clearly than could 3D TOF-MRA. ${ }^{22,23}$ However, in Silent MRA, the angiographic image is obtained by subtraction of images scanned before and after labeling. Thus, static tissue such as a thrombus cannot be detected in Silent MRA. ${ }^{22}$

In contrast to previous studies that found that MRA techniques were unable to provide a precise evaluation of the stent lumen, our study showed that diagnostic accuracy and the concordance rate between VWMRI and DSA were excellent. VWMRI is helpful in identifying the morphology of intracranial vessels. ${ }^{15}$ The present study found that VWMRI was more accurate than TOF-MRA for evaluating the status of the stented parent artery. VWMRI assisted the identification of 3 cases of stenosis and 1 of thrombosis, and its images demonstrated features similar to those shown by DSA.

In our preliminary study, VWMRI did not appear to be useful for evaluating aneurysm stability. We observed that in most cases, the SI of coiled aneurysms on VWMRI was heterogeneous regardless of follow-up duration. Thus, completeness of the coil embo- 
lization was overestimated by VWMRI. Various factors such as thrombus organization, fibrosis, and coil-related low SI seem to contribute to heterogeneous SI in the coiled aneurysm. TOFMRA (particularly with contrast) is good at detecting residual flow and aneurysm recurrence. It would seem that the techniques could be complementary.

The in-stent complication rates in our study were high. We observed that 3 of 4 patients were asymptomatic at follow-up. When a follow-up was performed with only TOF-MRA, DSA was recommended in cases of suspected abnormal findings with associated symptoms. In asymptomatic cases, follow-up was recommended but was often rejected by patients. However, after we used VWMRI, a subsequent DSA test was highly recommended and consequently less often rejected by patients. We speculate that this bias could be attributed to the high incidence of complications.

Asymptomatic in-stent stenosis can change into symptomatic stenosis, or spontaneous resolution of stenosis can occur. ${ }^{24}$ These events have important clinical implications for the continuation of dual-antiplatelet medication, close observation of neurologic symptoms, and follow-up. For clinical purposes, if MRA with or without contrast material depicts in-stent stenosis or stent occlusion, DSA should be performed to rule out in-stent stenosis or stent occlusion due to false-positive results. Our findings indicate that VWMRI provides a clear depiction of the patency of the parent artery. Accordingly, it is possible to avoid performing DSA in a group of patients in whom there are no particular clinical concerns for stent stenosis/occlusion. In practice, we believe that noninvasive imaging techniques such as VWMRI could be used more frequently than invasive conventional angiography as a follow-up technique.

This retrospective study has several limitations. First, follow-up MR imaging and DSA evaluations were not performed on the same day in most cases. Therefore, these 2 modalities might not reflect the exact same conditions in terms of the state of aneurysms and stented parent arteries. Second, intracranial stents were limited to the Neuroform and Solitaire varieties. Multiple flow diverters or flow-disruption devices are currently available for the treatment of cerebral aneurysms. Several series have reported the usefulness of MRA with contrast material in the follow-up of flow diverters or flow-disruption devices. ${ }^{25,26}$ These studies showed that contrast-enhanced MRA yielded better accuracy than TOF-MRA for the status of the parent artery. However, intraluminal evaluation remains difficult due to artifacts in MRA, regardless of the sequence used. This difficulty may be explained by the difference in composition but also porosity, metal coverage, and pore density between stents and flow diverters. ${ }^{25}$ The usefulness of VWMRI for variable flow diverters or flow-disruption devices should be determined in future studies.

Third, the goal of VWMRI is to reduce the intraluminal signal to zero. Intraluminal SI is not always suppressed fully on VWMRI. Visually, intraluminal SI appeared to be dark in our study. However, intraluminal signals were not zero. Thrombus was assumed if visual assessment revealed higher SI than intraluminal SI. However, there are limitations to visual evaluation when blood suppression is less effective in the presence of slow or complex flow. Novel blood-suppression techniques, including the Delay Alter- nating with Nutation for Tailored Excitation, can be used to reduce artifacts. ${ }^{27}$

Fourth, there are limitations concerning the TOF acquisition techniques. The SNR in the stent can be improved by a higher flip angle on the TOF technique. ${ }^{14}$ However, we did not use these techniques to improve image acquisition. With the optimization of MR imaging parameters of TOF-MRA according to the various intracranial stents, the luminal visualization of stents could be improved. We performed multislab TOF-MRA with multiple overlapping thin-slab acquisitions. The slab boundaries typically show some artifacts and intensity variations, even if acquired with a generous slab overlap. In our study, the slab artifacts did not significantly affect the reader's interpretation of the image quality. However, the slab boundary artifacts may reduce image quality if the stent is located directly in a slab boundary zone. The singleslab or sliding interleaved projection reconstruction technique can be used to reduce slab boundary artifacts over the conventional TOF technique. ${ }^{28}$

\section{CONCLUSIONS}

The present study performed quantitative and qualitative analyses of stented parent artery imaging using VWMRI and TOF-MRA. Even without contrast administration, VWMRI provided better image quality and higher accuracy in the evaluation of stented parent arteries than TOF-MRA. The high concordance rate of VWMRI for the analysis of pathologic parent artery status suggests that VWMRI could be useful in evaluating the patency of stented arteries after stent-assisted coiling.

\section{ACKNOWLEDGMENTS}

This work was supported by the Dong-A University Research Fund.

\section{REFERENCES}

1. Molyneux A, Kerr R, Stratton I, et al; International Subarachnoid Aneurysm Trial (ISAT) Collaborative Group. International Subarachnoid Aneurysm Trial (ISAT) of neurosurgical clipping versus endovascular coiling in 2143 patients with ruptured intracranial aneurysms: a randomised trial. Lancet 2002;360:1267-74 CrossRef Medline

2. Kemp WJ 3rd, Fulkerson DH, Payner TD, et al. Risk of hemorrhage from de novo cerebral aneurysms. J Neurosurg 2013;118:58-62 CrossRef Medline

3. Kim SR, Vora N, Jovin TG, et al. Anatomic results and complications of stent-assisted coil embolization of intracranial aneurysms. Interv Neuroradiol 2008;14:267-84 CrossRef Medline

4. King B, Vaziri S, Singla A, et al. Clinical and angiographic outcomes after stent-assisted coiling of cerebral aneurysms with Enterprise and Neuroform stents: a comparative analysis of the literature. J Neurointerv Surg 2015;7:905-09 CrossRef Medline

5. Schaafsma JD, Velthuis BK, Majoie CB, et al. Intracranial aneurysms treated with coil placement: test characteristics of follow-up MR angiography-multicenter study. Radiology 2010; 256:209-18 CrossRef Medline

6. Wang Y, Truong TN, Yen C, et al. Quantitative evaluation of susceptibility and shielding effects of nitinol, platinum, cobalt-alloy, and stainless steel stents. Magn Reson Med 2003;49:972-76 CrossRef Medline

7. Soize S, Gawlitza M, Raoult $\mathrm{H}$, et al. Imaging follow-up of intracranial aneurysms treated by endovascular means: why, when, and how? Stroke 2016;47:1407-12 CrossRef Medline 
8. The American College of Radiology. ACR Manual on Contrast Media. Version 10.3, 2018. https://www.acr.org/-/media/ACR/Files/ClinicalResources/Contrast_Media.pdf. Accessed August 29, 2018

9. Mandell DM, Mossa-Basha M, Qiao Y, et al; Vessel Wall Imaging Study Group of the American Society of Neuroradiology. Intracranial vessel wall MRI: principles and expert consensus recommendations of the American Society of Neuroradiology. AJNR Am J Neuroradiol 2017;38:218-29 CrossRef Medline

10. Bitar R, Leung G, Perng R, et al. MR pulse sequences: what every radiologist wants to know but is afraid to ask. Radiographics 2006; 26:513-37 CrossRef Medline

11. Takayama K, Taoka T, Nakagawa H, et al. Usefulness of contrastenhanced magnetic resonance angiography for follow-up of coil embolization with the Enterprise stent for cerebral aneurysms. J Comput Assist Tomogr 2011;35:568-72 CrossRef Medline

12. Park KJ, Jung SC, Kim HS, et al. Multi-contrast high-resolution magnetic resonance findings of spontaneous and unruptured intracranial vertebral artery dissection: qualitative and quantitative analysis according to stages. Cerebrovasc Dis 2016;42:23-31 CrossRef Medline

13. Willinsky RA, Taylor SM, TerBrugge K, et al. Neurologic complications of cerebral angiography: prospective analysis of 2,899 procedures and review of the literature. Radiology 2003;227:522-28 CrossRef Medline

14. Choi JW, Roh HG, Moon WJ, et al. Optimization of MR parameters of 3D TOF-MRA for various intracranial stents at 3.0T MRI. Neurointervention 2011;6:71-77 CrossRef Medline

15. Wong JH, Mitha AP, Willson M, et al. Assessment of brain aneurysms by using high-resolution magnetic resonance angiography after endovascular coil delivery. J Neurosurg 2007;107: 283-89 CrossRef Medline

16. Lövblad KO, Yilmaz H, Chouiter A, et al. Intracranial aneurysm stenting: follow-up with MR angiography. J Magn Reson Imaging 2006;24:418-22 CrossRef Medline

17. Thamburaj K, Cockroft K, Agarwal AK, et al. A comparison of magnetic resonance angiography techniques for the evaluation of intracranial aneurysms treated with stent-assisted coil embolization. Cureus 2016;8:e909 CrossRef Medline

18. Agid R, Willinsky RA, Lee SK, et al. Characterization of aneurysm remnants after endovascular treatment: contrast-enhanced MR angiography versus catheter digital subtraction angiography. AJNR Am J Neuroradiol 2008;29:1570-74 CrossRef Medline
19. Marciano D, Soize S, Metaxas G, et al. Follow-up of intracranial aneurysms treated with stent-assisted coiling: comparison of contrast-enhanced MRA, time-of-flight MRA, and digital subtraction angiography. J Neuroradiol 2017;44:44-51 CrossRef Medline

20. Akkaya S, Akca O, Arat A, et al. Usefulness of contrast-enhanced and TOF MR angiography for follow-up after low-profile stent-assisted coil embolization of intracranial aneurysms. Interv Neuroradiol 2018 Jan 1. [Epub ahead of print] CrossRef Medline

21. Choi JW, Roh HG, Moon WJ, et al. Time-resolved 3D contrastenhanced MRA on 3.0T: a non-invasive follow-up technique after stent-assisted coil embolization of the intracranial aneurysm. Korean J Radiol 2011;12:662-70 CrossRef Medline

22. Irie R, Suzuki M, Yamamoto M, et al. Assessing blood flow in an intracranial stent: a feasibility study of MR angiography using a silent scan after stent-assisted coil embolization for anterior circulation aneurysms. AJNR Am J Neuroradiol 2015;36:967-70 CrossRef Medline

23. Takano N, Suzuki M, Irie R, et al. Usefulness of non-contrast-enhanced MR angiography using a silent scan for follow-up after Yconfiguration stent-assisted coil embolization for basilar tip aneurysms. AJNR Am J Neuroradiol 2017;38:577-81 CrossRef Medline

24. Raymond J, Guilbert F, Weill A, et al. Long-term angiographic recurrences after selective endovascular treatment of aneurysms with detachable coils. Stroke 2003;34:1398-403 CrossRef Medline

25. Attali J, Benaissa A, Soize S, et al. Follow-up of intracranial aneurysms treated by flow diverter: comparison of three-dimensional time-of-flight MR angiography (3D-TOF-MRA) and contrast-enhanced MR angiography (CE-MRA) sequences with digital subtraction angiography as the gold standard. J Neurointerv Surg 2016;8: 81-86 CrossRef Medline

26. Patzig M, Forbrig R, Ertl L, et al. Intracranial aneurysms treated by flow-diverting stents: long-term follow-up with contrast-enhanced magnetic resonance angiography. Cardiovasc Intervent Radiol 2017; 40:1713-22 CrossRef Medline

27. Zhu C, Haraldsson H, Faraji F, et al. Isotropic 3D black blood MRI of abdominal aortic aneurysm wall and intraluminal thrombus. Magn Reson Imaging 2016;34:18-25 CrossRef Medline

28. Choi J, Seo H, Lim Y, et al. Sliding time of flight: sliding time of flight MR angiography using a dynamic image reconstruction method. Magn Reson Med 2015;73:1177-83 CrossRef Medline 